\title{
Dimensional preference changes as a function of overtraining
}

\author{
CHARLES L. RICHMAN \\ Wake Forest University, Winston-Salem, North Carolina 27109 \\ and \\ SUSAN SELVEY \\ West Virginia University, Morgantown, West Virginia 26506
}

\begin{abstract}
Preschool children were tested for their dimensional preferences prior to and following discrimination training. Children were administered either criterion level training or overtraining with their nondominant dimension relevant. The results showed: (1) Criterion level training failed to modify initial preferences when the dominant dimension was assessed to be "strong" or "weak"; (2) overtraining failed to modify initial preferences when the dominant dimension was assessed to be "strong"; (3) following overtraining with a "weak" dominant dimension, irrelevant initial preferences were modified in the direction of the initially nonpreferred dimension.
\end{abstract}

In most three-phased optional shift studies, Phase 1 consists of a variable-within problem involving at least one relevant and one irrelevant dimension. Following initial training, Phase 2 is immediately administered, whereby both dimensions (e.g., brightness and form) are relevant and redundant. During Phase 2, the task may be solved as a reversal or a nonreversal shift. In order to determine the basis of the subjects' selections, Phase 3 is initiated, in which test trials are interspersed with discrimination trials.

In experiments with young children, the usual finding is that reversal shifts are selected when and only when a strong dominant dimension is relevant during Phase 1 . If there is no dominant dimension or the dominant dimension is irrelevant, young children tend to make nonreversal or inconsistent choices (Kendler, 1974; Smiley, 1973). An exception to this finding is a study by Tighe and Tighe (1966), who showed that 4-year-old children selected reversal shifts when they were overtrained on their initially relevant nondominant dimension.

Taken together, these results suggest that: (1) Optional shift selections reflect preschool children's dimensional preferences, that is, dimensional preferences are not altered by criterion level discrimination training; (2) overtraining may alter children's preference for a particular dimension and, in so doing, modify the child's optional shift selection patterns.

This study was supported by Grant MH 21288-04 from the National Institute of Mental Health, PHS, awarded to Charles L. Richman. The manuscript was written while the first author was a Research Fellow at Harvard University and a Visiting Associate Professor at Tufts University. Reprint requests should be sent to Charles L. Richman, Department of Psychology, Wake Forest University, Winston-Salem, North Carolina 27109.
The present study was designed to test whether dimensional preferences are altered by discrimination training when cues on the nondominant dimension are initially (during Phase 1 training) relevant. Preschool children were either criterion trained or overtrained during Phase 1, with their nonpreferred dimension relevant. In addition, subjects were divided into twodimension preference groups, that is, "strong" and "weak." It was assumed that weak preferences would be easier to alter than strong ones.

\section{METHOD}

The subjects were 112 male and female $4 \frac{1}{2}$-year-old children ( \pm 4 months). The stimuli were four wooden forms; a black square, a white square, a black triangle, and a white triangle. A ping pong ball, the same brightness of the stimulus, was glued to the top of each stimulus.

Incomplete triad preference tests (Caron, 1970) were administered immediately prior to Phase 1 training and immediately following Phase 2 training. In the preference test, the child was asked which of the two test stimuli was "just like" the reference one. Form or brightness selections were recorded. All four possible stimulus combinations were tested twice, once each with the test stimuli on the left (right) side of the reference stimulus. A consistent eight out of eight responses classified the child as "strong dominant." Five to seven consistent responses classified the child as "weak dominant," and four consistent responses classified the child as "no preference."

Training according to the optional shift procedure involved Phases 1 and 2. In Phase 1, two pairs of stimuli were presented, one pair at a time, with order and position of the stimuli randomized. No pair was presented more than three consecutive times in the same position. All subjects were trained with their dominant dimension irrelevant. Subjects responded by pressing a button in front of one of the two stimuli. A noncorrection procedure was used. Criterion-trained (overtrained) children were required to reach a 10 out of 10 (30 out of 30 ) correct response criterion and were labeled Group 10/10 (Group 30/30). Children failing to obtain the 10 out of 10 correct response criterion within 75 trials were excused. 
During Phase 2, only one pair of stimuli was used and the value of the relevant dimension that was not rewarded during Phase 1 was now rewarded. Learning criterion was set at 10 out of 10 correct responses. For each correct response during Phase 1 and Phase 2 training, an $M \& M$ candy was given to the child; $M \&$ Ms were not given during pre- and post-Phase 1-2 preference testing.

\section{RESULTS AND DISCUSSION}

Of the 112 children tested, two were classified "no preference" and dropped from the study. Of the 110 children who showed a dimensional preference, $73 \%$ were classified "strong dominant" and $27 \%$ were "weak dominant" $\left[\chi^{2}(1)=22.72, p<.01\right]$. This finding is consistent with the results reported by Medin (1973).

The present results also showed that the preferred dimension of 68 (42) children was form (brightness) $\left[\chi^{2}(1)=6.25, p<.05\right]$. In order to equate the number of form- and brightness-dominant children in each group, 52 strong-dominant and 25 weak-dominant children were administered optional shift training. Of these children, one weak-dominant subject and 12 strong-dominant subjects failed to learn the Phase 1 task. Therefore, 24 weak-dominant and 40 strongdominant children completed the entire sequence of tests. Ten (14) of the weak-dominant children were criterion trained (overtrained) and 20 (20) of the strong-dominant children were criterion trained (overtrained).

A one-way analysis of variance performed on errors to the Phase 1 criterion for all subjects who were administered Phase 1 training revealed that the weakdominant children made fewer errors to the 10 out of 10 correct response criterion than did the strongdominant children; mean errors were 6.64 and 16.02, respectively $[F(1,75)=6.37, p<.05]$. The results confirmed the findings by Tighe, Tighe, Waterhouse, and Vasta (1970) that initial discrimination learning rates are correlated with dimensional preferences when preference tests are administered prior to testing. In fact, Tighe et al. (1970) have suggested that dimensional preference testing. may encourage the already existing preferences.

The primary purpose of this study was to determine whether discrimination training alters weak and/or strong dimensional preferences. To assess preference changes, each child's pretraining preference test score (number of responses to preferred dimension) was divided into his posttraining preference score, and that fraction was subtracted from 1.00 and multiplied by 100 , that is,

$$
\text { Percent Preference Change }=\left(1.00-\frac{\text { pretraining }}{\text { posttraining }}\right) \times(100 \%)
$$

The percent change scores for Groups 10/10 weak-dominant, $10 / 10$ strong-dominant, 30/30 weakdominant, and $30 / 30$ strong-dominant were $17 \%, 11 \%$, $61 \%$, and $19 \%$, respectively. A Kruskal-Wallis analysis of variance performed on the four groups' change scores showed statistically significant differences $[\mathrm{H}(3)=14.79$, $\mathrm{p}<.01]$. Subsequent Mann-Whitney U tests performed on all possible pairings of the four groups showed that the Group 30/30 weak-dominant change scores were significantly greater than those of Group 30/30 strongdominant, Group 10/10 strong-dominant, and Group $10 / 10$ weak-dominant $[\mathrm{z}=2.48, \mathrm{p}<.013 ; \mathrm{z}=3.10$, $\mathrm{p}<.002 ;$ and, $\mathrm{z}=2.24, \mathrm{p}<.025$, respectively]. All other pairwise comparisons were statistically nonsignificant (ps <.10). And, finally, individual Wilcoxin $T$ tests performed on each of the four groups' pre- vs posttraining dimensional preference scores showed that discrimination training failed to change initial preferences for children in Groups 30/30 strongdominant, 10/10 strong-dominant, and 10/10 weakdominant ( $p s>.10)$; however, the children trained in the $30 / 30$ weak-dominant condition chose the initially nonpreferred dimension more often in the post- than in the pretraining test $[\mathrm{T}(11)=5, \mathrm{p}<.05]$.

Taken together, the results indicate that discrimination training of the nonpreferred relevant dimension alters dimensional preferences when and only when training is extensive and initial preferences are weak. The present research is consistent with Smiley and Weir's (1966) dimensional dominance theory, which assumes prefered (nonpreferred) dimension learning produces reversal (nonreversal) choices. The present results suggest further that overtraining the nondominant dimension increases the child's preference for the nondominant dimension when preferences for the dominant dimension are weak, but not when they are strong. It is therefore assumed that optional reversal shift selections are facilitated by Phase 1 overtraining only if the weak-dominant dimension is irrelevant and/or the relevant dimension during Phase 1 is dominant. Tighe and Tighe (1966) showed that 4-yearold children failed to select reversal shifts when the nondominant dimension was initially relevant, unless they were overtrained; it is assumed that most of their overtrained children's dimensional preferences were relatively weak. Unfortunately, it is impossible to estimate preference strengths from the Tighe and Tighe (1966) report.

\section{REFERENCES}

Caron, A. J. Discrimination shifts in three-year-olds as a function of shift procedure. Developmental Psychology, 1970, 3, 236-241.

KENDLER, T. The effect of training stimulus variables on the reversal shift ontogency. Journal of Experimental Child Psychology, 1974, 17, 87-106.

Medin, D. L. Measuring and training dimensional preferences. Child Development. 1973, 44, 359-362. 
SMILEY, S. S. Optional shift behavior as a function of age and dimensional dominance. Journal of Experimental Child Psychology, 1973, 16, 451-458.

SMILEY, S. S., \& WeIR, M. W. Role of dimensional dominance in reversal and nonreversal shift behavior. Journal of Experimental Child Psychology, 1966, 4, 296-307.

Tighe, L. S., Tighe, T. J., Waterhouse, M. D., \& Vasta,
R. Dimensional preference and discrimination shift in children. Child Development, 1970, 41, 737-746.

Tighe, T. J., \& Tighe, L. S. Overtraining and optional shift behavior in rats and children. Journal of Comparative and Physiological Psychology, 1966, 62, 49-54.

(Received for publication November 1, 1976.) 